\title{
Back to basic, back to the future: searching for vital signals of life
}

\author{
Jubert Marquez ${ }^{1,2}$ - Maria Victoria Faith Garcia ${ }^{1,2}$ • Jin Han H,2 $^{1,2}$ \\ Received: 23 July 2020 / Revised: 23 July 2020 / Accepted: 6 August 2020 / Published online: 11 August 2020 \\ (C) Springer-Verlag GmbH Germany, part of Springer Nature 2020
}

There is a widening gap between research and clinical medicine. Translational medicine aims to bridge biomedical research toward tangible and practical hospital applications. Through the years, the term has gained wide usage as grantgiving bodies and researchers alike promise to transform the medical landscape with treatments that are least invasive, target-specific, time, and cost-efficient. Despite this goal, successful research-to-practice findings have continued to decline. Bench experiments focusing on small animal models, in vitro cell and tissue cultures, brought to light numerous mechanisms of disease that present potential treatment pathways, but results from large-scale studies have been disproportionate to the volume of basic science research produced [4]. A large percentage of studies fail in transitioning toward clinical use due to stringent requirements for ethical, regulatory, financial, and legal compliance. Part of the difficulty in transitioning from research to clinical practice may be attributed to laboratory methods that, though robust, are too simplistic to be applied to the complexities attributed to the human body.

Animal models and in vitro cell experiments have long been used to develop and establish medical therapies. The retrograde-perfused mammalian heart model by Oskar Langendorff is a vital modeling methodology that has remained robust since the time of its inception up until now, 125 years later. The Langendorff apparatus using murine heart remains relevant because of its replicability and simplicity while still providing vast and deep significance of studies

This article is a commentary to the original article https://oi.org/10.1007/ s00424-020-02446-6

Jin Han

phyhanj@inje.ac.kr

1 Department of Physiology, College of Medicine, Smart Marine Therapeutics Center, Cardiovascular and Metabolic Disease Center, Inje University, Busan 47392, Republic of Korea

2 Department of Health Sciences and Technology, BK21 Plus Project Team, Graduate School of Inje University, Busan 47392, Republic of Korea about and related to the physiology of the heart [2]. One major disadvantage of this model is the organismal discrepancy between the animal model and the human body. The use of large animal models, however, has produced sufficient outcomes with novel findings while maintaining ethical compliance which then translates toward feasible ethical regulations for human trials [3].

In this issue of Pflügers Archiv: European Journal of Physiology, Brook et al. reimagined the conventional ex vivo Langendorff apparatus to measure electrophysiological changes resulting from gap junction uncoupling in human and porcine hearts during carbenoxolone modulation. The study utilized porcine hearts and, resourcefully, used transplant-rejected human hearts to create a Langendorff apparatus for electrocardiographic study. Explanted human and porcine hearts were mounted and perfused in a custom-built Langendorff system which was connected to a system that records electrophysiological activity at 16 different myocardial sites. Carbenoxolone was administered to the hearts to simulate pro-arrhythmic conditions, as the drug has been demonstrated previously to reduce gap-junction coupling independent of ion channel regulation [1]. Individual recordings of the effects on the hearts were then recorded using contact electrogram and were analyzed and visualized for effects on cellular uncoupling. The paper puts forth an innovative solution to the question of how basic science research may translate toward substantial clinical applications.

Despite its promise, there are ethical and practical roadblocks that such a model needs to overcome before it can be further harnessed. As the authors have recognized as well, the use of an unhealthy human heart donor will exhibit a baseline much different from a healthy human and porcine heart despite carrying out correction and normalization calculations. Moreover, the use of porcine heart will not properly mimic the extensive remodeling a human heart during chronic ischemic disease even though it is widely used as it bears anatomical, morphological, and immune resemblance to the human heart [3]. And in order to perfect the use of a custom-made ex vivo model, numerous human and porcine hearts are need to be acquired, which will be made difficult by the strict ethical 
guidelines and regulations. Nonetheless, what Brook and colleagues have done here are nothing short of inventive. This setup has allowed Brook and his colleagues to perform analyses beyond the current limits of contractility and electrophysiological studies while maintaining a large stable heart ex vivo, something that previous researchers have had difficulty demonstrating before. Such a model, if further modified properly, can serve as a promising diagnostic tool that can assist clinicians and researchers alike with real-time analysis of arrhythmias in patients.

Funding information This research was supported by the Basic Research Lab Program (NRF2020R1A4A1018943) and the Basic Science Research Program (2018R1A2A3074998) through the National Research Foundation of Korea funded by the Ministry of Science and ICT, Korea.

\section{References}

1. Kojodjojo P, Kanagaratnam P, Segal OR, Hussain W, Peters NS (2006) The effects of carbenoxolone on human myocardial conduction: a tool to investigate the role of gap junctional uncoupling in human arrhythmogenesis. J Am Coll Cardiol 48:1242-1249. https:// doi.org/10.1016/j.jacc.2006.04.093

2. Milani-Nejad N, Janssen PM (2014) Small and large animal models in cardiac contraction research: advantages and disadvantages. Pharmacol Ther 141:235-249. https://doi.org/10.1016/j. pharmthera.2013.10.007

3. Spannbauer A, Traxler D, Zlabinger K, Gugerell A, Winkler J, Mester-Tonczar J, Lukovic D, Muller C, Riesenhuber M, Pavo N, Gyongyosi M (2019) Large animal models of heart failure with reduced ejection fraction (HFrEF). Front Cardiovasc Med 6:117. https://doi.org/10.3389/fcvm.2019.00117

4. Tageja N (2011) Bridging the translation gap - new hopes, new challenges. Fundam Clin Pharmacol 25:163-171. https://doi.org/ 10.1111/j.1472-8206.2010.00903.x

Publisher's note Springer Nature remains neutral with regard to jurisdictional claims in published maps and institutional affiliations. 